\title{
Prevalência de síndrome metabólica em pacientes curados de câncer pediátrico
}

\author{
Prevalence of metabolic syndrome in childhood cancer survivors
}

\author{
Barbara Saragiotto ${ }^{1}$, Gustavo Ribeiro Neves ${ }^{2}$, Marcelo Gil Cliquet ${ }^{3}$, \\ Marina Mayumi Murazawa ${ }^{4}$, Thiago Cardona Felipe ${ }^{5}$
}

Saragiotto B, Neves GR, Cliquet MG, Murazawa MM, Felipe TC. Prevalência de síndrome metabólica em pacientes curados de câncer pediátrico / Prevalence of metabolic syndrome in childhood cancer survivors. Rev Med (São Paulo). 2018 jan.-fev.;97(1):36-43.

RESUMO: O câncer atinge cerca de doze mil crianças por ano no Brasil. Parte delas, quando submetida a tratamento, tende a possuir maiores chances de desenvolver Síndrome Metabólica. Introdução: As crianças e adolescentes tratadas de câncer tendem a desenvolver a síndrome metabólica devido a alterações que os radioterápicos e quimioterápicos propiciam. Essas disfunções acometem os tecidos: adiposo, osteomuscular, endotelial; o trato gastrointestinal e o fígado. Objetivos: Analisar a prevalência de síndrome metabólica em crianças curadas de câncer e comparar as variáveis estudadas. Materiais e Métodos: Avaliamos trinta e oito pacientes do Hospital GPACI que tenham terminado o tratamento de câncer há, no mínimo, cinco anos. Foram investigadas variáveis como: glicemia, pressão arterial, circunferência abdominal, HDL, triglicerídeos, tipo de câncer, tipo de tratamento e histórico familiar. Como grupo controle, utilizamos 20 irmãos dos pacientes analisados sob os mesmos parâmetros. Resultados: Sobreviventes tiveram maior chance de desenvolver síndrome metabólica (RR 2,1 Mid-p 0,27). Tanto no grupo controle quanto no grupo de curados, o HDL foi o índice que teve mais alterações, estando abaixo ou igual a $45 \mathrm{mg} / \mathrm{dL}$ em $46 \%$ dos voluntários. Comparando o grupo estudado com o grupo controle, a circunferência abdominal foi o fator de maior relevância (RR 5,2 Mid-p 0,02).

\begin{abstract}
Discussão: Contrastamos a prevalência de síndrome metabólica encontrada nos curados de câncer tanto com seus irmãos quanto com crianças e adolescentes dos Estados Unidos da América. Em ambas as comparações, a prevalência no grupo estudado foi maior, o que é semelhante a outras pesquisas realizadas. Conclusão: A circunferência abdominal foi o fator que apresentou significância estatística e maior risco de acometer os sobreviventes de câncer infantil. Diferentemente de outros estudos, os outros índices não tiveram significância estatística, provavelmente pelo tamanho da amostra.
\end{abstract}

Descritores: Neoplasias/radioterapia; Neoplasias/terapia; Neoplasias/tratamento farmacológico; Glicemia; HDL-colesterol; Doenças metabólicas; Criança.

ABSTRACT: In Brazil, cancer affects around twelve thousand children per year. Many of them when submitted to a treatment have increased chances to develop metabolic syndrome when compared to another child. Introduction: Adolescents and children treated of cancer are propitious to develop metabolic syndrome due to changes that radiotherapy and chemotherapy provides. These dysfunctions compromise fat tissue, muscle, skeletal,

1. Pontifícia Universidade Católica de São Paulo - PUCSP, Faculdade de Ciências Médicas e da Saúde de Sorocaba, Sorocaba, SP, BR. Aluna de Iniciação Científica. Email: ba saragiotto@hotmail.com. Orcid: 0000-0002-3172-8761

2. Hospital GPACI. Co-orientador de Iniciação Científica. Email: gustavoneo@bol.com.br. Orcid: 0000-0002-2580-6970

3. Pontifícia Universidade Católica - PUCSP, Faculdade de Ciências Médicas e da Saúde de Sorocaba, Sorocaba, SP, BR. Orientador de Iniciação Científica. Email: mmcliquet@uol.com.br. Orcid: 0000-0002-4990-6677

4. Pontifícia Universidade Católica - PUCSP, Faculdade de Ciências Médicas e da Saúde de Sorocaba, Sorocaba, SP, BR. Email: marina. murazawa@gmail.com. Orcid: 0000-0002-7431-0912

5. Pontifícia Universidade Católica - PUCSP, Faculdade de Ciências Médicas e da Saúde de Sorocaba, Sorocaba, SP, BR. Email: thiagocardona21@gmai.com. Orcid: 0000-0002-3837-8987

Endereço para correspondência: Rua Albina Barbosa, 219. São Paulo, SP. CEP: 01530-020. 
Saragiotto B, et al. Prevalência de síndrome metabólica em pacientes curados de câncer pediátrico.

endothelium tissue, gastrointestinal tract and the liver. Objectives: Evaluate the prevalence of metabolic syndrome in children treated of cancer and compare with parameters investigated. Materials and methods: Thirty-eight patients of GPACI Hospital that had finished cancer treatment in five years at least were analyzed. It was considered variations such as: plasma glucose, blood pressure, waist circumference, HDL, triglycerides, cancer type, treatment type and familiar historical. As control group, we used 20 siblings of the analyzed patients under the same parameters. Results: Survivors had increased risk to develop metabolic syndrome (RR 2,11 Mid-p 0,27). HDL was the index with most alterations in control group and in the studied group (less or equal $45 \mathrm{mg}$ / dLin $46 \%$ of volunteers). Comparing the studied group with the

\section{INTRODUÇÃO}

$\mathrm{O}$ câncer infantil corresponde, no Brasil e em grande parte do mundo, à primeira causa de morte por doença entre pacientes de 1 a 19 anos $^{1}$. Segundo o Instituto Nacional de Câncer, a incidência em 2012 foi de 11.530 pacientes, sendo que, nos últimos 30 anos, o progresso no tratamento do câncer infantil deu um salto impressionante: se diagnosticado precocemente e tratado em centros especializados, a taxa de sobrevida é de $70 \%{ }^{1}$.

As melhores taxas de sobrevida dessas crianças com diagnóstico de câncer trouxeram consigo o reconhecimento de efeitos clinicamente significativos a longo prazo, consequentes ao tratamento. Estimativas sugerem que até $90 \%$ dos pacientes curados de câncer na infância terão um transtorno de saúde crônico até os 45 anos. Estudos recentes têm mostrado que esses pacientes remanescentes de câncer pediátrico tem um risco aumentado para síndrome metabólica em relação a população em geral - adiposidade visceral, hipertensão, dislipidemia, resistência a insulina -, podendo desenvolver uma doença cardiovascular prematura $^{2,3,4,5}$.

Uma compreensão abrangente da prevalência e dos mecanismos patológicos da síndrome metabólica em sobreviventes de câncer infantil é crucial para o desenvolvimento de intervenções diretas para limitar as implicações a longo prazo na saúde destes indivíduos.

Este conjunto de anormalidades afeta vários sistemas de órgãos envolvidos no metabolismo de lipídeos e hemostasia glicêmica, entre eles os tecidos ósseo, muscular, adiposo, endotelial, o trato gastrointestinal e o fígado ${ }^{2,6,7}$.

No tecido ósseo temos a osteocalcina como chave do metabolismo, esta que consiste em uma proteína produzida pelos osteoblastos cuja função, além da homeostase do cálcio, é a de aumentar a secreção de insulina através da estimulação da proliferação de células beta. A combinação da quimioterapia e da radioterapia reduz em grande escala o número e a atividade dos osteoblastos, levando a redução da produção de osteocalcina e uma consequente resistência insulínica que contribui para o desenvolvimento da obesidade ${ }^{2}$.

Sobreviventes de câncer pediátrico apresentam control group, the waist circumference was the most important factor (RR 5,2 Mid-p 0,02). Discussion: As seen in other reports, the prevalence of metabolic syndrome in the group survivors of pediatric cancer was superior to the control group and also higher than the prevalence of metabolic syndrome in children and adolescents of USA. Conclusion: Waist circumference was statiscally significant with a RR 5,2 (mid-p 0,02). Differently from other studies, the other factors were not relevant, probably because the sample size.

Keywords: Neoplasms/radiotherapy; Neoplasms/therapy; Neoplasms/drug therapy; Blood glucose; Cholesterol, HDL; Metabolic diseases; Child.

ainda destruição da integralidade dos microtúbulos e do citoesqueleto, resultando em uma menor captação e transporte de glicose, aumentando a chance desses pacientes se tornarem diabéticos tipo $2^{2,4}$.

$\mathrm{O}$ câncer por si próprio inicia a primeira onda de alterações no tecido adiposo, estas que são ampliadas pelo tratamento, resultando numa disfunção do adipócito e diminuição da secreção da adiponectina - regula diretamente o metabolismo da glicose, além de intermediar na função endotelial e na aterosclerose. A inflamação sistêmica pelo câncer e seu tratamento podem, a longo prazo, ter impacto em distúrbios metabólicos pela disfunção do tecido adiposo, que persiste mesmo após o fim da exposição à quimioterapia - as adipocinas, particularmente as segregadas a partir de adipócitos viscerais, são prejudiciais ao endotélio vascular, criando um ambiente favorável ao desenvolvimento de placa.

Por sua vez, a lesão endotelial dos vasos leva ao desenvolvimento de doenças cardíacas, tanto pela formação de placas de ateroma (aterosclerose), quanto por inflamação e fibrose das células endoteliais (arteriosclerose) $)^{8}$. A quimioterapia afeta diretamente o endotélio devido a sua citotoxicidade, levando a apoptose das células endoteliais danificadas. Outro mecanismo de lesão pelos terapêuticos é a diminuição da produção de óxido nítrico pelo endotélio, levando a rigidez vascular e consequentemente hipertensão. Há ainda fármacos usados para tratar as neoplasias que levam ao bloqueio dos receptores VEGF, culminando em inflamação e invasão de células espumosas, causando aterosclerose e agravando o risco cardiovascular já existente nessa população ${ }^{2,3,4}$.

No trato gastrointestinal de pacientes tratados de câncer, agentes quimioterápicos tem impacto direto na alteração da flora intestinal, levando a reconstituição dela com um fenótipo diferente após a destruição e contribuindo, assim, para uma menor absorção calórica, maior resistência insulínica e obesidade ${ }^{2}$.

Vários agentes quimioterápicos causam doença hepatocelular provocando mudanças na gliconeogênese e no transporte de glicose. Isso ocorre devido à alteração do transportador, da expressão e da translocação de glicose, que levam a hiperglicemia. A lesão hepatocelular também 
leva a diminuição da capacidade da oxidação dos ácidos graxos no fígado, provocando dislipidemia ${ }^{2}$.

Outros estudos apontam para uma disfunção endócrina induzida por radiação crânio-espinhal, que leva a deficiência do hormônio de crescimento (GH). Danos ao eixo hipotálamo-hipófise pela irradiação crânioespinhal seriam altamente responsáveis pela diminuição da concentração de $\mathrm{GH}$ a longo termo, provavelmente por uma desregulação dos hormônios hipotalâmicos. Ademais a deficiência de GH é fortemente associada a reatividade vascular anormal e desenvolvimento de aterosclerose, e também a um padrão de dislipidemia e obesidade abdominal. A deficiência de GH também foi determinada em sobreviventes de câncer pediátrico tratados apenas com quimioterapia, o que pode ser explicado por uma combinação da susceptibilidade individual com a capacidade dos quimioterápicos ultrapassarem a barreira hematoencefálica ${ }^{2,4}$.

A síndrome metabólica representa a interseção dos sistemas endócrino e cardiovascular, uma relação que é particularmente complexa em sobreviventes de câncer infantil.

Desta forma, tais pacientes formam um grupo de risco distinto para síndrome metabólica, uma vez que acumulam uma série de alterações teciduais as quais prejudicam o metabolismo como um todo ${ }^{9,10}$. Esta síndrome encontra-se presente quando o paciente possui três dos seguintes critérios:

$\checkmark$ HDL menor que 40mg/dl em homens e 50mg/ dl em mulheres;

$\checkmark$ Triglicerídeos acima de $150 \mathrm{mg} / \mathrm{dl}$;

$\checkmark$ Glicemia de $110 \mathrm{mg} / \mathrm{dl}$ ou diabetes estabelecido;

$\checkmark$ Hipertensão arterial: Pressão sistólica maior que $130 \mathrm{mmHg}$ e/ou diastólica maior que $85 \mathrm{mmHg}$;

$\checkmark$ Circunferência abdominal maior que $88 \mathrm{~cm}$ em mulheres e $102 \mathrm{~cm}$ em homens.

A síndrome metabólica é bastante estudada em adultos, no entanto, em crianças é de pouco conhecimento ${ }^{11}$. Não há grandes estudos brasileiros demonstrando a prevalência de síndrome metabólica em crianças. Assim, levamos em consideração estudos americanos que abordam essa particularidade, bem como estudos brasileiros sobre obesidade e má alimentação em crianças. Além disso, tal síndrome está cada dia mais presente nessa faixa etária, variando de $4,2 \%$ até $9,2 \%{ }^{12-18}$.

\section{JUSTIFICATIVA}

A decisão sobre a realização deste projeto foi baseada na escassez de informações e pesquisas sobre o assunto no Brasil. Além disso, seu desenvolvimento configura-se como uma ótima oportunidade para rastrear futuros portadores de doenças crônicas relevantes tais como diabetes, hipertensão arterial, hipercolesterolemia e síndrome metabólica, bem como estabelecer correlações com o tratamento de câncer pediátrico que geram adultos de risco. Diante disso, ainda seria possível desenvolver intervenções terapêuticas e fornecer aconselhamento de saúde direcionado a esse grupo de pessoas a fim de diminuir a prevalência dessas complicações.

\section{OBJETIVOS}

\section{Primários}

$\checkmark$ Avaliar a prevalência de síndrome metabólica em pacientes curados de câncer infanto-juvenil.

$\checkmark$ Avaliar a associação entre síndrome metabólica e variáveis relacionadas à neoplasia e o tratamento utilizando os irmãos como grupo controle.

$\checkmark$ Comparar a prevalência de síndrome metabólica dos pacientes avaliados com a de crianças, adolescentes e adultos em geral.

\section{Secundários}

$\checkmark$ Relacionar o tipo de tratamento (quimioterapia e/ ou radioterapia) realizado com a presença de alterações na pressão arterial, glicemia, triglicérides, colesterol HDL e circunferência abdominal.

$\checkmark$ Analisar a prevalência de síndrome metabólica nos dois grupos, pacientes curados de câncer e irmãos desses, estatisticamente.

\section{METODOLOGIA}

Para a execução deste trabalho, foi realizada uma pesquisa quantitativa na qual foram recrutados via telefone 38 pacientes de ambos os sexos, já acompanhados no ambulatório do Hospital GPACI, curados de câncer infantil há mais de cinco anos e admitidos entre os anos de 1995 e 2010, para avaliação dos critérios de diagnóstico da síndrome metabólica. Foram aplicados os mesmos critérios para os irmãos(ãs) desses pacientes, também recrutados via telefone, os quais funcionaram como grupo controle foram atribuídos como tal uma vez que se submetem aos mesmos fatores de risco (genética, nível socioeconômico, localização geográfica etc.). Sua participação foi voluntária, anônima e respaldada pelo termo de consentimento livre esclarecido. Pacientes que não atenderam aos critérios de inclusão citados acima, não foram considerados.

Foram avaliadas as seguintes medidas em ambulatórios realizados semanalmente no Hospital GPACI: (1) Circunferência abdominal; (2) Pressão arterial; (3) Glicemia de jejum; (4) Triglicérides; (5) Colesterol HDL; (6) Histórico Familiar; (7) Tipo de Câncer e (8) Tipo de tratamento.

$\checkmark$ Para avaliação da circunferência abdominal, foi tomado como base as medidas propostas pela Federação Internacional de Diabetes (IDF) de grande fidedignidade e fácil acesso, segundo a qual é descrita em percentil, em 
Saragiotto B, et al. Prevalência de síndrome metabólica em pacientes curados de câncer pediátrico.

que é considerada como alta acima do percentil 90.

$\checkmark$ Utilizou-se o estudo "Second Task Force of Blood Pressure in Children, 1987", atualizado pelo "National High Blood Pressure Education Program" em 2004, como forma de avaliar a pressão arterial dos pacientes. O estudo utilizou como critério de pré hipertensão e hipertensão valores acima do percentil 90 e 95 respectivamente ${ }^{16}$.

$\checkmark$ Foi considerado como alteração da glicemia os valores acima de $110 \mathrm{mg} / \mathrm{dL}$, para todas as idades, já que esse é o preconizado pela maioria dos estudos e pela Federação Internacional de Diabetes.

$\checkmark$ Com relação ao HDL e triglicerídeos, foi considerado significativo para todas as idades o HDL menor ou igual a $45 \mathrm{mg} / \mathrm{dL}$ e os triglicerídeos maior ou igual a $130 \mathrm{mg} / \mathrm{dL}$, de acordo com a I Diretriz Brasileira para a Prevenção da Aterosclerose na Infância e na Adolescência cuja se adequa melhor ao perfil dos pacientes estudados ${ }^{19}$.

Todas as informações coletadas foram organizadas em uma tabela comparativa entre o grupo estudado e o grupo controle. Por fim, houve um balanço estatístico para definir quais variáveis (idade, sexo, tipo de neoplasia) tiveram maior relevância no risco de desenvolvimento da Síndrome Metabólica em pacientes curados de câncer. Além disso, foram analisadas as frequências: pressão arterial, glicemia, triglicerídeos, colesterol HDL, histórico familiar (diabetes mellitus, hipertensão arterial e doenças cardíacas) e suas correlações com as doenças de base.

Estabeleceu-se como histórico familiar, parentes (pai, mãe, irmãos, avós) portadores de diabetes mellitus, hipertensão arterial e acidentes cardiovasculares (infarto agudo do miocárdio ou acidente vascular encefálico).

Decidiu-se por não utilizar o peso e IMC dos pacientes por não serem parte dos critérios de síndrome metabólica.

\section{RESULTADOS}

A análise estatística foi feita pela ferramenta Open Source Epidemiologic Statistics for Public Health ${ }^{20}$, por permitir a realização de diversos cálculos e comparações. Utilizou-se o Mid-p quando a análise tinha como resultados positivos um número inferior ou igual a 5 , e o qui quadrado quando fosse maior, de acordo com o recomendado pelo site e pela literatura ${ }^{20,21}$.

Inicialmente, identificou-se os pacientes que se encaixavam nos critérios de inclusão pré-definidos, como: ter desenvolvido câncer na infância, ter finalizado o tratamento há mais de 5 anos e ter sido admitido entre os anos de 1995 e 2010. Foi então desenvolvida uma ficha de atendimento para triagem dos pacientes, contendo todas as informações necessárias para o projeto e utilizada nos encontros com o grupo estudado, que possuía: nome, registro hospitalar $(\mathrm{RH})$, data de nascimento, data de admissão e data de fim do tratamento, diagnóstico (dentre eles - LLA, LMA, neuroblastoma, tumor cerebral, sarcoma, rabdomiossarcoma, tumor de Ewing, tumor de Wilms, tumor de células germinativas, linfoma de Hodgkin, linfoma não-Hodgkin, outros); realização ou não de radioterapia; realização ou não de quimioterapia; histórico familiar para Diabetes Mellitus, Hipertensão Arterial ou doença cardiovascular. Além do já citado, incluiu-se a medida da circunferência abdominal, da pressão arterial e os resultados laboratoriais de glicemia, triglicerídeos e HDL, colhidos pela equipe de enfermagem do Hospital GPACI e analisados pelo laboratório do mesmo hospital. Tais dados foram obtidos tanto dos pacientes do grupo estudado quanto de seus irmãos (quando possuíam), esses atribuídos como grupo controle.

Foi então, explicitado o desenvolvimento e objetivo do trabalho, convidando-os a participar, o que era formalizado pela assinatura do TCLE (no caso dos maiores de 18 anos) e do Termo de Assentimento e Termo de Responsabilidade (no caso de menores de 18 anos e seus pais, respectivamente).

A construção de tabelas com os resultados obtidos foi realizada, dividindo-se os pacientes num gráfico por número de alterações correspondentes a síndrome metabólica Hipertensão, Diabetes mellitus, Hipertrigliciridemia, HDL baixo e circunferência abdominal elevada - de acordo com seus devidos grupos, estudado e controles (Figuras 1 e 2). Foram recrutados no total 58 indivíduos via telefone, sendo 38 pacientes como grupo estudado (ex-portadores de câncer infantil) sendo a média de idade 15,2 anos, e 20 pacientes como grupo controle (voluntários irmãos), com a média de idade 17,2 anos.

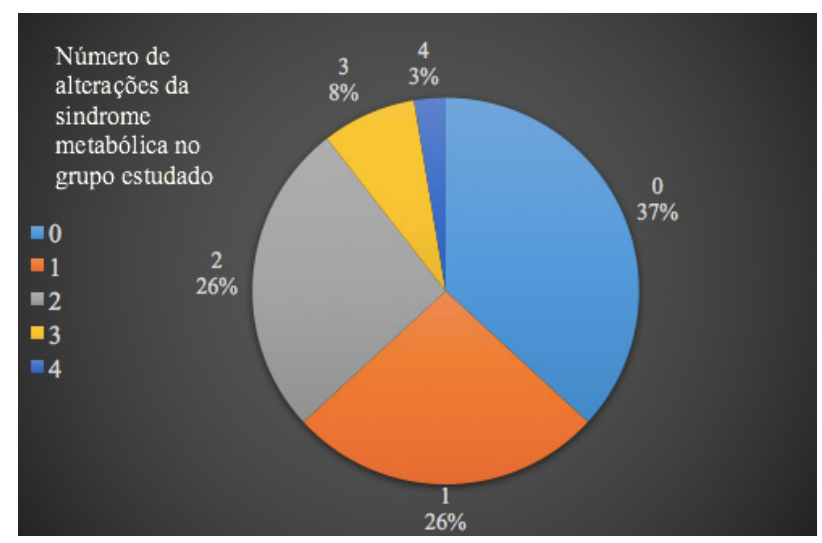

Figura 1. Número de alterações da síndrome metabólica por paciente no grupo curados de câncer 


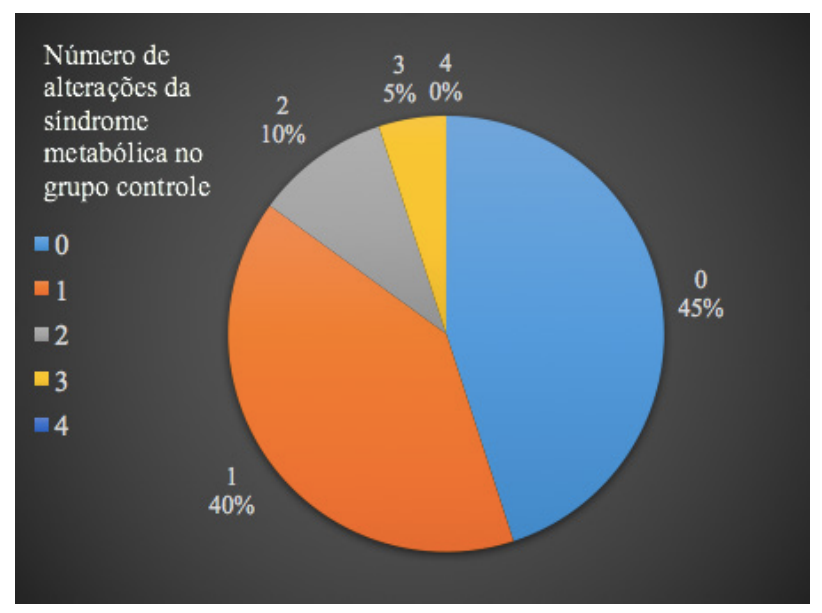

Figura 2. Número de alterações da síndrome metabólica por paciente no grupo controle

No que diz respeito a escassez do grupo controle, estudou-se a possibilidade de comparar os resultados obtidos dos pacientes curados de câncer pediátrico com a literatura, no caso de a amostra de irmãos ser pequena e insuficiente para comparação e obtenção de resultados relevantes.

Foram diagnosticados 4 pacientes $(10,5 \%)$ com síndrome metabólica entre os pacientes que tiveram neoplasia na infância e 1 paciente $(5 \%)$ com síndrome metabólica no grupo controle, obtendo-se risco relativo de 2,11 (Mid-p = 0,27). A presença de alteração do HDL esteve presente em 28 voluntários dos 58 estudados, sendo o critério mais prevalente. Já a glicemia, foi o menos adulterado com apenas 3 dos 58 pacientes avaliados. Por sua vez, o critério circunferência abdominal foi o único que apresentou relevância estatística, apresentando risco relativo de 5,2 (Mid-p = 0,02). Outro fator que apresentou risco importante foi a pressão arterial, porém não sendo possível o cálculo, já que não se obteve alterações no grupo controle.

Em relação ao tipo de tratamento, pode-se concluir que 14 deles realizaram quimioterapia associada a radioterapia, enquanto que 24 realizaram apenas uma das modalidades acima referidas (vinte e dois pacientes somente quimioterapia e dois pacientes somente radioterapia). Portanto, comparando-se os tipos de tratamento utilizados - nas modalidades monoterapia (radioterapia ou quimioterapia) e terapia conjunta (radioterapia e quimioterapia) -, foi possível observar que 3 dos pacientes diagnosticados com Síndrome Metabólica utilizaram as duas terapias, enquanto que apenas 1 realizou monoterapia, revelando um risco relativo de 5,14 (Mid-p $=0,07)$.

Ainda realizando essa comparação, houve maior predomínio de critérios positivos nos pacientes que realizaram a dupla terapia, em relação aos que efetuaram a monoterapia. Os triglicerídeos apresentaram um risco relativo de 5,14 (Mid-p = 0,01 IC=1,19-22,1) do grupo submetido a terapia combinada em relação ao grupo tratado em terapia isolada.

No que diz respeito ao tipo de câncer encontrado, observou-se a frequência de Leucemia linfoide aguda $(\mathrm{n}=20)$, Leucemia mieloide aguda/ Linfoma de Hodgkin/ Neuroblastoma $(\mathrm{n}=4)$, e em menor número, Meduloblastoma $(\mathrm{n}=2)$, Rabdomiossarcoma/ Fibromatose/ Sarcoma Osteogenico/ Tumor de Ewing ( $\mathrm{n}=1$ ).

Tabelas referentes aos resultados da pesquisa.

Tabela 1: Critérios alterados em cada sexo dos pacientes curados de câncer

\begin{tabular}{l|c|c|c|c|c}
\hline Sexo & $\begin{array}{l}\text { Circunferência } \\
\text { abdominal }\end{array}$ & Glicemia & Pressão arterial & Triglicerídeos & HDL \\
\hline Masculino & 3 & 0 & 3 & 2 & 9 \\
\hline Feminino & 7 & 3 & 1 & 6 & 9 \\
\hline Total & 10 & 3 & 4 & 8 & 18 \\
\hline
\end{tabular}

Tabela 2: Número de parâmetros alterados em cada sexo dos pacientes curados de câncer

\begin{tabular}{l|c|c|c|c|c}
\hline Sexo & 0 Alteração & 1 Alteração & 2 Alterações & 3 Alterações & 4 Alterações \\
\hline Masculino & 7 & 8 & 2 & 0 & 1 \\
\hline Feminino & 7 & 3 & 7 & 3 & 0 \\
\hline Total & 14 & 11 & 9 & 3 & 1 \\
\hline
\end{tabular}


Saragiotto B, et al. Prevalência de síndrome metabólica em pacientes curados de câncer pediátrico.

Tabela 3: Alterações em cada grupo por critério de síndrome metabólica (Total de 38 pacientes no grupo de pacientes que tiveram câncer e 20 no grupo controle

\begin{tabular}{l|c|c|c|c|c}
\hline Critérios & $\begin{array}{l}\text { Número de } \\
\text { alterados no grupo } \\
\text { estudado }\end{array}$ & $\begin{array}{l}\text { Número de } \\
\text { alterados no grupo } \\
\text { controle }\end{array}$ & Total de alterações & Risco relativo & $\begin{array}{l}\text { Teste estatístico e } \\
\text { resultado }\end{array}$ \\
\hline $\begin{array}{l}\text { Circunferência } \\
\text { abdominal }\end{array}$ & 10 & 1 & 11 & 5,2 & Mid-p 0,02 \\
\hline Triglicerídeos & 8 & 4 & 12 & 1,05 & Mid-p 0,47 \\
\hline HDL & 18 & 10 & 28 & 0,94 & Qui-quadrado 0,42 \\
\hline Pressão arterial & 4 & 0 & 4 & Indefinido & Mid-p 0,08 \\
\hline Glicemia & 2 & 1 & 3 & 1,05 & Mid-p 0,49 \\
\hline
\end{tabular}

Tabela 4: Número de pacientes com alteração em cada grupo e a razão de risco em relação a terapia com quimioterapia e radioterapia

\begin{tabular}{l|c|c|c|c}
\hline & Monoterapia & Terapia combinada & Razão de risco & Mid-p \\
\hline HDL & 11 & 7 & 1,09 & 0,40 \\
\hline Pressão arterial & 3 & 1 & 0,57 & 0,33 \\
\hline Glicemia & 1 & 2 & 3,42 & 0,17 \\
\hline $\begin{array}{l}\text { Circunferencia } \\
\text { abdominal }\end{array}$ & 5 & 5 & 1,7 & 0,17 \\
\hline Triglicerídeos & 2 & 6 & 5,14 & 0,01 \\
\hline
\end{tabular}

(No presente estudo: 24 realizaram monoterapia e 14 dupla terapia)

\section{DISCUSSÃO}

Foi observado através deste estudo que os indivíduos sobreviventes de câncer infantil, principalmente os submetidos ao tratamento combinado de quimioterapia e radioterapia, possuem maior risco de desenvolver componentes da síndrome metabólica (razão de risco maior que um em quatro dos cinco critérios), assim como descrito na literatura ${ }^{22}$. Comparando este trabalho com os outros estudos, a prevalência de síndrome metabólica foi menor, o que pode ser devido à faixa etária dos voluntários avaliados (Tabela 5). Outro aspecto que pode ter influenciado na prevalência encontrada nos diferentes estudos é a falta de padronização dos critérios avaliados e de seus pontos de corte.

Tabela 5: Visão geral de estudos sobre a síndrome metabólica em pacientes sobreviventes de câncer infantil.

\begin{tabular}{l|c|l|c|c}
\hline Referência & $\mathbf{N}^{\mathbf{1}}$ & Diagnósticos & $\begin{array}{l}\text { Idade média em anos } \\
\text { (variação) }\end{array}$ & $\begin{array}{l}\text { Prevalência de síndrome } \\
\text { metabólica }\end{array}$ \\
\hline Trimis $^{23}$ & 80 & Variados & $13.9(5.2-24.1)$ & $11 \%$ \\
\hline Gurney $^{24}$ & 75 & Variados & 30 & $16.6 \%$ \\
\hline Hoffman $^{25}$ & 32 & Sarcoma & $37.2(17.5-55.4)$ & $33 \%$ \\
\hline Taskinen $^{26}$ & 31 & Tratados com transplante de células tronco & $15(7-34)$ & $39 \%$ \\
\hline Van Wass $^{27}$ & 466 & Variados & $27.6(18.2-59.1)$ & $12.6 \%$ \\
\hline Cliquet & 38 & Variados & $15.2(7-30)$ & $10,5 \%$ \\
\hline
\end{tabular}


Saragiotto B, et al. Prevalência de síndrome metabólica em pacientes curados de câncer pediátrico.

Em três estados norte-americanos (Texas, Carolina do Norte e Califórnia) foi realizado um estudo de prevalência de Síndrome Metabólica em adolescentes randomizados sem histórico de câncer infantil. Nesse estudo, 138 participantes de 1.453 foram classificados com síndrome metabólica (aproximadamente 9,49\%) ${ }^{28}$. Já um trabalho brasileiro com 582 adolescentes randomizados e também sem história prévia de neoplasia pediátrica, apresentou uma prevalência de $6,7 \%$ de síndrome metabólica ${ }^{29}$. Correlacionando com este estudo realizado no hospital GPACI, o qual obteve prevalência de síndrome metabólica de 10,5\%, é possível observar maiores índices nos pacientes curados de câncer. Isso pode ser associado aos efeitos dos tipos de quimioterápicos e radioterápicos utilizados no tratamento com os efeitos adversos metabólicos e endocrinológicos (lesão tecidual, deficiência de $\mathrm{GH})$.

Em outras referências literárias e trabalhos científicos, foi demonstrado a presença de apenas alguns critérios isolados alterados da síndrome metabólica ${ }^{24,27,30}$. No presente estudo isso também foi demonstrado, com relação à circunferência abdominal e à pressão arterial.

Em um estudo holandês com 466 sobreviventes de câncer pediátrico, $13 \%$ deles tiveram dois componentes da síndrome metabólica alterados, enquanto no trabalho apresentado o número no grupo de pacientes curados de neoplasia pediátrica foi de $26 \%$. Apenas $3 \%$ dos pacientes holandeses tiveram três critérios positivos e somente uma pessoa obteve quatro parâmetros alterados. Já no presente estudo, foi obtido $8 \%$ com três parâmetros positivos e também somente um indivíduo com quatro critérios.

Foi observado nesta pesquisa realizada no Hospital GPACI, que dos quatro pacientes portadores de síndrome metabólica no grupo curado de câncer, três eram mulheres e um homem. E dos 18 pacientes que apresentaram HDL alterado, nove eram mulheres e nove homens, o que difere do estudo realizado por Waas et al. ${ }^{22}$, em que os homens

\section{REFERÊNCIAS}

1. Instituto Nacional de Câncer José Alencar Gomes da Silva (INCA). Tipos de câncer: infantil [citado 23 jan. 2018]. Disponível em: http://www2.inca.gov.br/wps/wcm/connect/ tiposdecancer/site/home/infantil.

2. Rosen GP, Nguyen HT, Shaibi GQ. Metabolic syndrome in pediatric cancer survivors: A mechanistic review. Pediatr Blood Cancer. 2013;60(12):1922-8. doi: 10.1002/pbc.24703.

3. van Waas VM, Neggers SJ, van der Lelij AJ, Pieters R, van den Heuvel-Eibrink MM. Metabolic syndrome in adult survivors of childhood cancer, a review. J Pediatr Hematol Oncol. 2010;32(3):171-9. doi: 10.1097/MPH.0b013e3181d419c3.

4. Meacham RL, Chow EJ, Ness KK, Kamdar KY, Chen Y, Yasui Y, Oeffinger KC, Sklar CA, Robison LL, Mertens AC. Cardiovascular risk factors in adult survivors of pediatric cancer - a report from the childhood cancer survivor study. tiveram HDL maior do que as mulheres. Apesar disso, os outros resultados obtidos desta pesquisa são semelhantes ao de Waas et al. ${ }^{22}$. Não houve diferenças significativas comparando-se incidência de dois ou mais critérios e relacionando-os com o sexo dos pacientes.

\section{CONCLUSÃO}

Pode-se concluir principalmente que a quimioterapia e a radioterapia levam ao surgimento de alteraçôes metabólicas e endocrinológicas para a população estudada. A hipótese é que esses tratamentos provavelmente levam a um aumento da resistencia insulínica, dificuldade de captação e transporte de glicose, lesão endotelial (resultando em aterosclerose), aumento da resistência vascular, aumento da adiposidade visceral, deficiência do hormônio de crescimento $(\mathrm{GH})$ entre outros fatores.

Ademais, pode se inferir que a quimioterapia combinada com a radioterapia é mais agressiva do que a terapia isolada (quimioterapia ou radioterapia), uma vez que foram obtidos mais resultados positivos para síndrome metabólica em pacientes tratados com ambas terapêuticas $(21,4 \%)$ diante dos tratados com monoterapia (4,1\%).

A patogênese da síndrome metabólica em sobreviventes de câncer infantil é amplamente especulativa, e possivelmente as interações entre o estilo de vida (por exemplo inatividade física, desnutrição, tabagismo), tipo de tratamento e fatores ambientais contribuem para o agrupamento de anormalidades metabólicas.

A síndrome metabólica representa a interseção dos sistemas endócrino e cardiovascular, uma relação que é particularmente complexa em sobreviventes de câncer infantil. A pesquisa focada na compreensão dos mecanismos prejudiciais da síndrome metabólica em sobreviventes de câncer infantil é crucial para orientar o desenvolvimento de intervenções direcionadas para melhorar ou prevenir os resultados vasculares adversos.

Cancer Epidemiol Biomarkers Prevent. 2010;19:170-81. doi: 10.1158/1055-9965.EPI-09-0555.

5. Abu-Ouf NM, Jan MM. Metabolic syndrome in the survivors of childhood acute lymphoblastic leukaemia. Obes Res Clin Pract. 2015;9(2):114-24. doi: 10.1016/j.orcp.2014.06.004.

6. de Haas EC, Oosting SF, Lefrandt JD, Wolffenbuttel BH, Sleijfer DT, Gietema JA. The metabolic syndrome in cancer survivors. Lancet Oncol. 2010;11(2):193-203. doi: 10.1016/ S1470-2045(09)70287-6.

7. Ehrhardt MJ, Mulrooney DA. Metabolic syndrome in adult survivors of childhood cancer: the intersection of oncology, endocrinology, and cardiology. Lancet Diabetes Endocrinol. 2015;3(7):494-6. doi: 10.1016/S2213-8587(15)00031-5.

8. Meacham LR. Pediatric cancer survivors at risk for diseases that predispose them to heart disease. American Association 
Saragiotto B, et al. Prevalência de síndrome metabólica em pacientes curados de câncer pediátrico.

for Cancer Research; January10, 2010.

9. Nelson R. Diabetes in cancer survivors a late effect of abdominal radiation? Medscape.2016 Jan19. Available from: http://www.medscape.com/viewarticle/857405.

10. Meacham LR,Sklar CA, Li S, Liu Q, Gimpel N, et al. Diabetes mellitus in long-term survivors of childhood cancer: increased risk associated with radiation therapy a report for the Childhood Cancer Survivor Study (CCSS). Arch Intern Med. 2009;169(15):1381-8. doi: 10.1001/ archinternmed.2009.209.

11. Pergher NQR, Melo ME, Halpern A, Mancini MC. O diagnóstico de síndrome metabólica é aplicável às crianças? J. Pediatr (Rio J.). 2010;86(2):101-8. http://dx.doi.org/10.2223/ JPED.1983.

12. Speiser PH, Rudolf MCJ, Anhalt H, Camacho-Hubner C, Chiarelli F, et al. Consensus Statement: Childhood obesity. J Clin Endocrinol Metab. 2005;90:1871-87. https://doi. org/10.1210/jc.2004-1389.

13. Hedley AA, Ogden CL, Johnson CL, Carrol MD, Curtin LR, Flegal KM. Prevalence of overweight and obesity among US children, adolescents, and adults, 1999-2002. JAMA. 2004;291:2847-50. doi: 10.1001/jama.291.23.2847.

14. Cook S, Weitzman M, Auinger P, Nguyen M, Dietz WH. Prevalence of a metabolic syndrome phenotype in adolescents. Arch Pediatr Adolesc Med 2003;157:821-7. doi: 10.1001/archpedi.157.8.821.

15. Ferranti SD, Gauvreau K, Ludwig DS, Neufeld EJ, Newburger JW, Rifai N. Prevalence of the metabolic syndrome in American adolescents: findings from the Third National Health and Nutrition Examination Survey. Circulation. 2004; 110:2494-7. doi: 10.1161/01.CIR.0000145117.40114.C7.

16. dos Santos AAC, Zanetta DMT, Cipullo JP, Burdmann EA. O diagnóstico da hipertensão arterial na criança e no adolescente. Pediatria (São Paulo) 2003;25(4):174-83. Disponível em: http://www.luzimarteixeira.com.br/wpcontent/uploads/2010/08/pa-em-criancas-e-adolesc.pdf.

17. Ford ES, Li C, Zhao G, Pearson WS, Mokdad AH. Prevalence of the metabolic syndrome among U.S. adolescents using the definition from the International Diabetes Federation. Diabetes Care. 2008;31:587-9. doi: 10.2337/dc07-1030.

18. Duncan GE, Li SM, Zhou XH. Prevalence and trends of a metabolic syndrome phenotype among U.S. adolescents, 1999-2000. Diabetes Care. 2004;27:2438-43. Available from: http://care.diabetesjournals.org/content/27/10/2438.long.

19. I Diretriz para a Prevenção da Aterosclerose na Infância e na Adolescência. Arq Bras Cardiol. 2005;85(Suppl 6):4-36. http://dx.doi.org/10.1590/S0066-782X2005002500001.

20. Dean AG, Sullivan KM, Soe MM. OpenEpi: open source epidemiologic statistics for public health. Available from: www.openepi.com.
21. Berry G, Armitage P. Mid-p confidence intervals: a brief review. J R Statistical Soc. Series D (The Statistician). 1995;44(4):417-23. doi: 10.2307/2348891.

22. van Waas V, Neggers SJ, van der Lelij AJ, Pieters R, van den Heuvel-Eibrink MM. Metabolic syndrome in adult survivors of childhood cancer, a review. J Pediatr Hematol Oncol. 2010;32(3):171-9.doi: 10.1097/MPH.0b013e3181d419c3.

23. Trimis G, Moschovi M, Papassoriou I, Chrousos G, Tzortzatou-Stathopoulou F. Early indicators of dysmetabolic syndrome in young survivors of acute lymphoblas $c$ leukemia in childhood as a target for preventing disease. J Pediatr Hematol Oncol. 2007;29:309-14. doi: 10.1097/ MPH.0b013e318059c249

24. Gurney JG, Ness KK, Sibley SD, O’Leary M, Dengel DR, Lee JM, Youngren NM, Glasser SP, Baker KS. Metabolic syndrome and growth hormone defciency in adult survivors of childhood acute lymphoblastic leukemia. Cancer. 2006;107:1303-12. doi: 10.1002/cncr.22120.

25. Hoffman KE, Derdak J, Bernstein D, Reynolds JC, Avila NA, Gerber L, Steinberg SM, Chrousos G, Mackall CL, Mansky PJ. Metabolic syndrome traits in long-term survivors of pediatric sarcoma. Pediatr Blood Cancer 2008;50:341-6. doi: 10.1002/pbc.21363.

26. Taskinen M, Lipsanen-Nyman M, Tii nen A, Hovi L, Saarinen-Pihkala UM. Insu cient growth hormone secre on is associated with metabolic syndrome a er allogeneic stem cell transplantation in childhood. J Pediatr Hematol Oncol. 2007;29:529-34.

27. van Waas M, Neggers SJ, Pieters R, van den Heuvel-Eibrink MM. Components of the metabolic syndrome in 500 adult long-term survivors of childhood cancer. Ann Oncol 2010;21:1121-6. doi: 10.1093/annonc/mdp414.

28. Jago R. Prevalence of the metabolic syndrome among a racially/ethnically diverse group of u.s. eighth-grade adolescents and associations with fasting insulin and homeostasis model assessment of insulin resistance levels. Diabetes Care. 2008;31(10):2020-5. doi: 10.2337/dc08-0411.

29. Stabelini Neto A, Bozza R, Ulbrich A, Mascarenhas LPG, Boguszewski MCS, Campos W. Síndrome metabólica em adolescentes de diferentes estados nutricionais. Arq Bras Endocrinol Metab. 2012;56(2):104-9. http://dx.doi. org/10.1590/S0004-27302012000200003.

30. Gunn HM, Emilsson H, Gabriel M, Maguire AM, Steinbeck KS. Metabolic health in childhood cancer survivors: a longitudinal study in a long-term follow-up clinic. J Adolesc Young Adult Oncol. 2016;5(1):24-30. doi: 10.1089/ jayao.2015.0036.

Recebido em: 04.10.16

Aceito em: 06.02.18 